\title{
Halo removal experiments with hollow electron lens in the BNL Relativistic Heavy Ion Collider
}

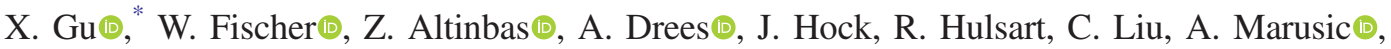 \\ T. A. Miller, M. Minty, G. Robert-Demolaize, Y. Tan $\odot$, and P. Thieberger $\odot$ \\ Brookhaven National Laboratory, Upton, New York 11973, USA \\ H. Garcia Morales $\oplus^{\dagger}$ \\ CERN, 1211 Geneva, Switzerland and Royal Holloway, University of London, \\ Egham, TW20 OEX, United Kingdom \\ D. Mirarchi, ${ }^{\ddagger}$ S. Redaelli, and A. I. Pikin® \\ CERN, 1211 Geneva, Switzerland \\ G. Stancariø \\ Fermilab, Batavia, Illinois 60510, USA
}

(Received 22 November 2019; accepted 10 February 2020; published 9 March 2020)

\begin{abstract}
A hollow electron beam has been proposed as an active control tool to remove the beam halo from highenergy, high-current hadron or ion machines (such as the High-Luminosity Large Hadron Collider). To study the halo removal rate and assess the effect on the ion beam core, one of the two electron lenses in the Relativistic Heavy Ion Collider was changed from a Gaussian beam profile to a hollow profile. We describe the design and verification of the hollow electron beam parameters as well as the methods to minimize the hollow beam profile distortions, which can result in an ion beam emittance increase. The hollow beam alignment with the ion beam by using a backscattered electron detector has been demonstrated. Furthermore, experiments were carried out to explore the efficiency of the halo removal by scanning the current and inner radius of the hollow electron beam, which is pulsed either every turn or every nth turn. The effects of the hollow electron beam on the ion beam emittance and luminosity were also assessed experimentally by scanning the inner radius of the electron beam.
\end{abstract}

DOI: 10.1103/PhysRevAccelBeams.23.031001

\section{INTRODUCTION}

Hollow electron beams have been studied for about 70 years for different applications. Nonmagnetized hollow electron beams have been used or proposed for several acceleration schemes, such as in linear induction accelerators [1] and, more recently, plasma wakefield accelerators [2-3]. Furthermore, to improve the stability and performance of a free-electron laser, an electron beam with a hollow transverse distribution instead of the usual Gaussian is under investigation [4]. To get better transverse cooling

\footnotetext{
*xgu@bnl.gov

Present address: CERN and University of Oxford, Oxford OX1 2JD, United Kingdom.

*Also at University of Manchester, Manchester, M13 9PL, United Kingdom.

Published by the American Physical Society under the terms of the Creative Commons Attribution 4.0 International license. Further distribution of this work must maintain attribution to the author(s) and the published article's title, journal citation, and DOI.
}

rates and avoid electron heating, as well as to increase the ion beam lifetime, a magnetized hollow electron beam has been proposed and used for an electron cooler [5-11].

More recently, hollow electron lenses were proposed as a novel technique for active beam collimation and halo control with application for the High-Luminosity Large Hadron Collider (HL-LHC) at CERN [12-15]. Concerns related to the operation with high stored beam energies at the HL-LHC motivated studies for the integration of this technique in the baseline upgrade program of the LHC. A first conceptual design [13] was followed by detailed integration studies [15].

The hollow electron beam can be used as an active control method to remove the beam halo via increasing the diffusion rate within a certain amplitude range, which results in a higher collimation efficiency. This reduces the beam energy stored between the beam core and the collimator edges and reduces the risk of a sudden energy release from the beam halo, by controlling the moments in time during the operational cycle when beams are lost. The beam halo is sensitive to both beam condition changes, such as orbit jitters, and 
machine configuration changes, such as tune adjustments, optics changes (squeeze), collimator position movements, and fast crab cavity parameter changes.

The technique was first tested experimentally at the Fermilab Tevatron collider [16-19]. During these experiments, the same electron-lens current was seen by the antiprotons at each turn (the so-called "dc mode" of operation). More studies have followed, such as the calculation of the transverse kicks from the hollow electron-lens bends [20], the beam diocotron instability control [21], and the hollow electron gun characterization [22], as well as experimental and numerical studies for a hollow electron-lens system [23-28]. The above studies, as well as the existing electron-lens experience from Tevatron and RHIC [29-34], provide information for the design of a hollow electron-lens system that meets the halo removal requirements for the HL-LHC, as well as the future applications of beam collimation in the FCC-hh [35] at CERN or Super Proton-Proton Collider (SPPC) [36] in China.

Following the conceptual design of hollow electron lenses for beam halo control in the LHC, the preliminary mechanical design and simulation study of hollow electron beam collimation in the HL-LHC has been finished. Meanwhile, the effect of the pulsed hollow beam on the proton beam core (emittance), the halo population, and the halo removal rate in the LHC were simulated in detail $[24,25]$. In the above study, in order to obtain a higher halo removal rate, two different pulsing patterns instead of the dc mode are considered: a random mode and a resonant mode. For the random mode, the electron beam is pulsed randomly. For the resonant mode, the electron beam is turned on every nth turn.

For the pulsed mode, there are concerns about potential emittance growth due to electron beam profile distortions, field asymmetries between the entrance and the exit bending region of the hollow electron-lens system, and beam alignment errors. There currently is an effort ongoing to improve the beam transverse profile via simulation by reducing the hollow electron beam diocotron instability [21].
To find a method to reduce the hollow electron beam profile distortions and to study the halo removal rate with different operating configurations, as well as testing a better method for the hollow beam alignment, the yellow ring electron lens in the Relativistic Heavy Ion Collider (RHIC) was modified from providing a Gaussian electron beam to a hollow electron beam.

The RHIC consists of two rings on a common horizontal plane: the blue ring for clockwise and the yellow ring for counterclockwise beams. The two RHIC electron lenses (e-lenses) with Gaussian transverse profiles, both located near the interaction point (IP) IP10, were designed to compensate for the beam-beam effects from the protonproton interactions at the two interaction points IP6 and IP8 [29-34].

The paper is organized as follows. In Sec. II, the RHIC electron-lens system is briefly described. In Sec. III, the design considerations and characteristics of the hollow electron beam are presented. In Sec. IV, the methods to reduce the hollow beam distortions are discussed. In Sec. V, the alignment between a hollow electron beam and a hadron beam is demonstrated using a backscattered electron detector [37]. Results of dedicated beam-beam experiments with the hollow electron beam are presented in Sec. VI. A summary and discussion are given in Sec. VI.

\section{RHIC ELECTRON-LENS SYSTEM}

Figure 1 is a schematic horizontal sectional view of one of the two RHIC electron lenses [33]. Each RHIC electron lens includes an electron gun and a collector [38] as the electron beam source and dump. They are on the same side, which forms a $U$ shape along with the superconducting solenoid (Fig. 1), while the LHC design plans an S-shape layout with the gun and collectors at opposite sides to selfcompensate edge effects seen by the proton beam at the entrance and exit of the electron beam. It also has magnets [39-40] for beam trajectory control and beam size modulation. There is instrumentation [41-42] for the beam diagnostic and the alignment of the electron and ion beams.

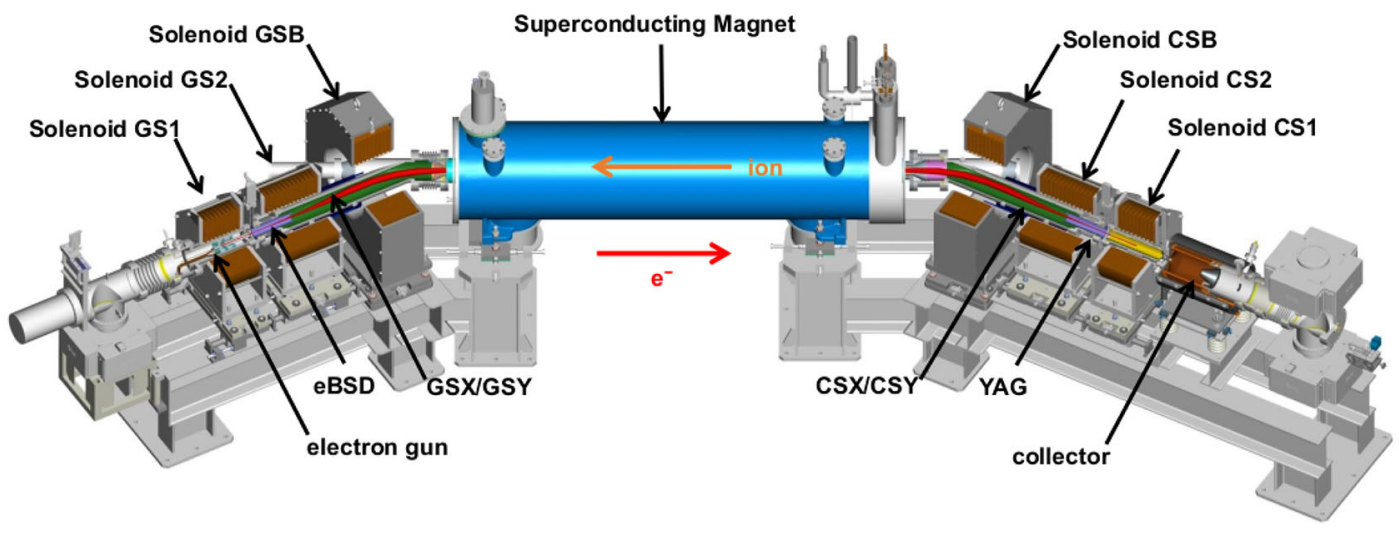

FIG. 1. Schematic layout of one of the RHIC electron lenses [33]. 
TABLE I. Main electron and ion beam parameters for the RHIC hollow lens experiments.

\begin{tabular}{lcccc}
\hline \hline Parameter & Unit & \multicolumn{2}{c}{ Value } \\
\hline Ion beam parameters & & $\mathrm{Ru}(2018)^{\mathrm{a}}$ & $\mathrm{Au}(2018)^{\mathrm{a}}$ & $\mathrm{Au}(2019)^{\mathrm{a}}$ \\
Total ion beam energy $E_{\text {ion }}$ & $\mathrm{GeV}$ /nucleon & 100 & 13.5 & 90 \\
Relativistic factor $\gamma_{\text {ion }}$ & & 107.5 & 14.5 & 96.8 \\
$\beta^{*}{ }_{x, y}$ at IP10 & $\mathrm{m}$ & 5.0 & 7.0 & 5.0 \\
rms emittance $\varepsilon_{n}$ & $\mathrm{~mm} \mathrm{mrad}$ & 1.5 & 2.0 & 2.0 \\
rms beam size at IP10, $\sigma^{*}{ }_{p}$ & $\mathrm{~mm}$ & 0.26 & 0.98 & 0.32 \\
Electron-lens parameters & & & & 4.0 \\
Gun, inner radius $r_{g 1}$ & $\mathrm{~mm}$ & 4.0 & 5.5 & 5.5 \\
Gun, outer radius $r_{g 2}$ & $\mathrm{~mm}$ & 5.5 & 1.0 & 3.0 \\
Main solenoid field & $\mathrm{T}$ & 3.0 & $0.14-0.56$ & $0.14-0.56$ \\
Gun solenoid field & $\mathrm{T}$ & $0.14-0.56$ & $1.50-3.00$ & $0.86-1.73$ \\
E-lens, inner radius b & $\mathrm{mm}$ & $0.86-1.73$ & $2.06-4.12$ & $1.19-2.38$ \\
E-lens, outer radius d & $\mathrm{mm}$ & $1.19-2.38$ & 12.7 & 12.7 \\
Beam pipe radius at the gun & $\mathrm{mm}$ & 12.7 & 54.6 & 54.6 \\
Beam pipe radius in the solenoid & $\mathrm{mm}$ & 54.6 & & \\
\hline \hline
\end{tabular}

${ }^{\mathrm{a}} 2018$ and 2019 are the years for the experiments.

As shown in Fig. 1, the electron beam is emitted from the electron gun, which is immersed in the magnetic field of the gun-side solenoid GS1. Then it goes through the gun-side warm solenoids GS2 and GSB and propagates through the superconducting solenoid magnet in which the interaction with the hadron beam takes place. This is followed by another set of three solenoids, CSB, CS2, and CS1, that is symmetric with the gun-side solenoids, and the beam is absorbed in the collector. The combined fields of the GSB and CSB and the superconducting magnet as well as the four dipole correctors (GSX, GSY, CSX, and CSY) are used for the beam trajectory control during the beam propagation.

The transverse beam radius of a hollow electron beam inside of magnets can be calculated as

$$
r=r_{\text {cathode }} \sqrt{\frac{B_{\text {cathode }}}{B_{0}}},
$$

where $r$ and $r_{\text {cathode }}$ are the beam radius of the hollow electron beam in the magnet and at the cathode, respectively, while $B_{0}$ and $B_{\text {cathode }}$ are the magnetic fields during propagation and at the cathode, respectively.

For the electron beam profile measurement, there is a yttrium-aluminum-garnet (YAG) screen located between CS2 and CS1. There are two beam position monitors (BPMs) installed inside the superconducting magnet for the initial electron beam alignment with the hadron beam. For the final beam alignment, the backscattered electron detector [37] is used, which is located between GS1 and GS2 above the electron beam.

Lists of RHIC hollow electron gun and beam parameters, as well as the ion parameters in the experiments, are given in Table I. Ion beam parameters are listed for the $100 \mathrm{GeV} /$ nucleon $\mathrm{Ru}$ (ruthenium) and 13.5 and $90 \mathrm{GeV} /$ nucleon $\mathrm{Au}$ (gold) beams.

\section{HOLLOW ELECTRON GUN}

\section{A. Hollow cathode design}

To have a hollow electron beam in one of the RHIC electron lenses, one of two existing Gaussian electron guns was modified. To reduce the risks and cost of the modification, the existing control electrode, anode, and cathode holder remained unchanged. These were designed for a 7.5mm-radius cathode. The newly designed hollow cathode has a $4 \mathrm{~mm}$ inner radius and $5.5 \mathrm{~mm}$ outer radius.

To reduce the possibility of a diocotron instability at the edges, the cathode geometry was optimized with the 2D package Tricomp from Field Precision [43]. The final design is shown in Fig. 2. The left schematic shows the hollow gun components, electrostatic field, and beam trajectory. The optimization procedure was done with the RCDS code [44]. The optimization reduced the total oscillation amplitude of the outermost and innermost beam trajectories. The right plot shows the oscillation amplitude of the outermost trajectory. At the end of the optimization, the oscillation amplitude of the outermost trajectory was reduced from the beginning of the optimization. Each line between them represents one simulation.

A dispenser cathode (a porous tungsten matrix impregnated with a barium-based emission-enhancing material) was selected as the hollow cathode material [45].

\section{B. Beam current and Pierce instability}

The gun perveance $P_{\text {gun }}$ gives the current for a certain anode voltage expressed by Child's law [46-49]

$$
I_{e}=P_{\mathrm{gun}} \cdot U_{\text {anode }}^{3 / 2},
$$



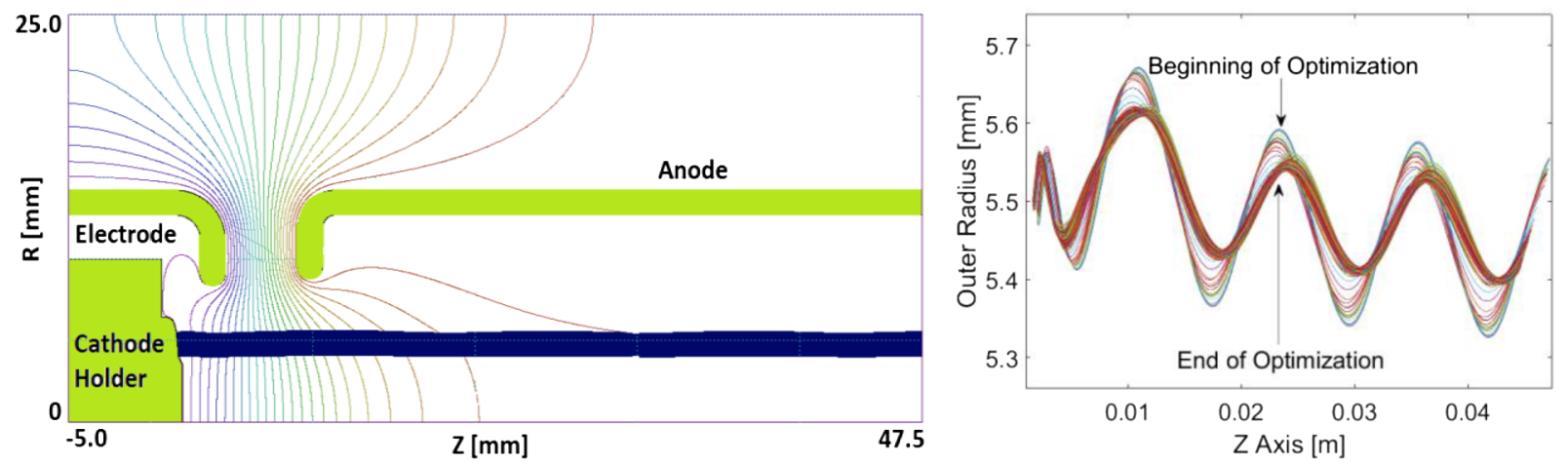

FIG. 2. Simulated hollow cathode geometry (left) and trajectory optimization with simulation (right).

where $I_{e}$ is the electron beam current and $U_{\text {anode }}$ is the anode potential difference to the cathode. The gun perveance is determined by the geometry.

The perveance of the RHIC hollow electron gun was measured in pulsed mode (Fig. 3, left). A fit to Eq. (2) gives $P_{\text {gun }}=1.15 \pm 0.01 \mu \mathrm{AV}^{-3 / 2}$. A maximum current of 1.5 A was drawn from the hollow gun.

The drift space perveance and the Pierce instability threshold are other characteristics of the machine setup, which can be used to determine the minimum required beam energy or the maximum current for electron beam propagation without space charge or virtual cathode effects. Any current exceeding this maximum value will result in the formation of a virtual cathode and reflection of a part of the electron beam.

For a nonrelativistic low-energy beam propagating through a vacuum chamber, it is assumed that the beam is accelerated in a stationary electric field. In this case, the drift space perveance can be written as

$$
I_{e, \max }=P_{\mathrm{drift}} \cdot U_{\mathrm{drift}}^{3 / 2},
$$

where $U_{\text {drift }}$ is the potential difference between the drift tube (or vacuum chamber) and the cathode and $I_{e, \max }$ is the maximum current that can propagate through the drift tube.

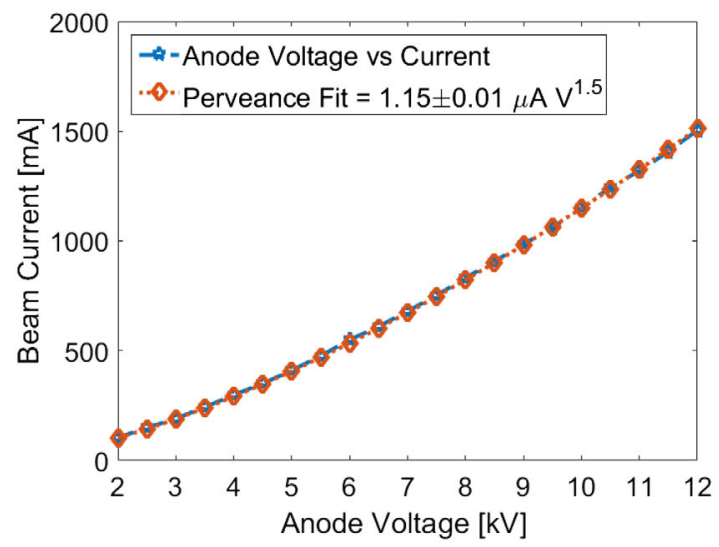

In the case of the Brillouin hollow beam, where the particles move with a constant radius, the drift space perveance can be expressed as (see Ref. [49], p. 289)

$$
P_{\mathrm{drift}}=\frac{1+b^{2} / d^{2}}{1-b^{2} / d^{2}} \cdot \frac{16 \pi \varepsilon_{0}}{3 \sqrt{6}} \cdot \sqrt{\frac{e}{m}} \cdot \frac{1}{1+2 \ln (R / d)},
$$

where $b, d$, and $R$ are the hollow beam inner radius, the outer radius, and the vacuum pipe radius, respectively. Equation (4) can be used to estimate the minimum energy needed for a specified current limit, with the constraints of beam pipe radius and cathode geometry.

To measure the drift space perveance and the Pierce instability threshold, the maximum current was recorded as a function of the beam energy until the noise on the scraper is detectable [31]. Two signals in the RHIC e-lens were used as indicators for the maximum current and the formation of a virtual cathode. They were the noise on the waveform signal from the scrapers (collector side) [31] and the anode (gun side).

This maximum current is plotted as a function of the beam energy in Fig. 3 (right) for a hollow electron beam. After fitting, the measured drift space perveances are $3.35 \pm 0.10, \quad 3.65 \pm 0.05, \quad$ and $4.01 \pm 0.04 \mu \mathrm{AV}^{-3 / 2}$,

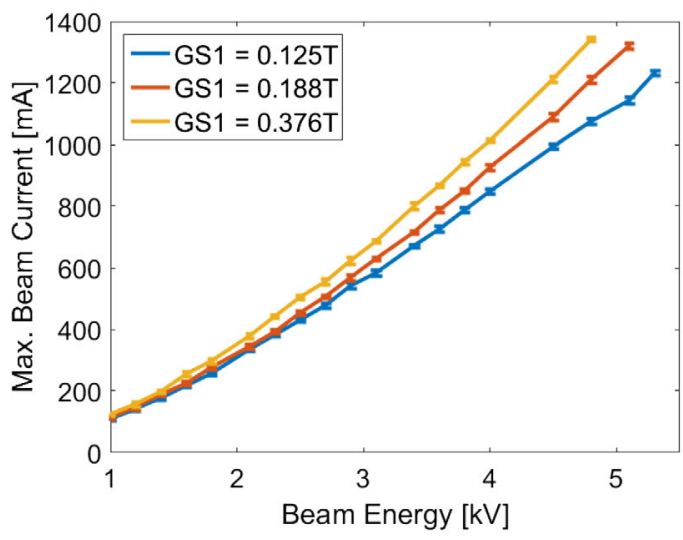

FIG. 3. Electron gun perveance measurement for a hollow electron beam (left) and measurement of drift space perveance with different GS1 magnetic fields (right). 
respectively, for $0.125,0.188$, and $0.376 \mathrm{~T}$ GS1 magnetic field.

For the case of the RHIC hollow electron lens, the conditions for Brillouin flow are not exactly satisfied because of a strong magnetic field modulation. But from Eq. (4) it is found qualitatively that increasing the GS1 magnetic field increases the drift space perveance, since it increases the beam size during beam propagation. This has been demonstrated for Gaussian beams as reported in Ref. [31] and verified here again with the hollow electron beam experimentally.

\section{HOLLOW BEAM DISTORTIONS}

\section{A. Theoretical studies}

In beam halo control with a pulsed hollow electron beam, one of the main concerns is the possibility of ion beam emittance growth. For an ideal radially symmetric hollow electron lens with an S-shaped geometry and perfect alignment, the beam core would experience a zero-net kick.

In reality, there are three primary deviations from this ideal case. The first is the beam merger region, where the ion beam crosses the electron beam edge and gets inside of the hollow electron beam. This has been discussed in Refs. $[20,26]$. The second is the misalignment between the hollow electron beam and the ion beam, which will be addressed in Sec. V. The third is a distorted transverse hollow electron beam profile, which will be discussed below.

For an ideal radially symmetric hollow electron beam, there is a zero-net force. In the presence of a hollow beam profile distortions, in the central region overlapped to the beam core, the force is nonzero and could possibly cause ion beam emittance growth.

Distortions of a hollow electron beam profile can stem from cathode surface defects, misalignment during gun installation, and the asymmetry of the bending magnetic field during propagation, as well as the so-called diocotron instability. The diocotron instability can be the dominating factor for the distortions if the other effects are minimized.

In 1955, it was discovered experimentally that a hollow electron beam could break up into discrete current bundles during propagation within a magnetic field [50]. The term "diocotron instability" was then coined in the following theoretical studies [51-54] to describe this instability, which is analogous to the Kelvin-Helmholtz phenomenon in fluid dynamics. It is driven by the shear motion of the beam when both an external magnetic field and the selfelectric field are present.

For the hollow electron beam without an inner inductor [55], the angular velocity can be simplified as (see Ref. [55], p. 298)

$$
\omega(r)=\omega_{D}(r) \cdot\left(1-\frac{b^{2}}{r^{2}}\right) .
$$

Equation (5) indicates that the beam angular velocities are different at different radii, which results in the relative motion of different beam layers. In Eq. (5), $b$ is the inner radius of the electron beam, while $\omega_{D}(r)$ is the effective diocotron frequency for the electrons defined [55] as

$$
\omega_{D}(r)=\frac{\omega_{p e}^{2}}{2 \omega_{c e}}=\frac{e \cdot n_{e}(r)}{2 \epsilon_{0} \gamma^{2} B_{0}},
$$

where $n_{e}(r)$ is the electron density of the hollow electron beam while $B_{0}$ is the longitudinal magnetic field.

The electron gun for the hollow electron beam is immersed in a magnetic field through which the electron beam is propagated. Therefore, according to the adiabatic theorem of magnetic flux conservation, $B \cdot \Delta S=$ const [49], and, provided there is no current loss during propagation, the electron beam density inside the electron-lens system can be written as

$$
\frac{n_{e}(r)}{B_{0}}=\frac{n_{\text {cathode }}(r)}{B_{\text {cathode }}},
$$

where $n_{\text {cathode }}(r)$ and $B_{\text {cathode }}$ are the electron density and the magnetic field at the cathode, respectively. The effective diocotron frequency of the electrons, which is determined only by the electron density and the magnetic field at the cathode, is finally expressed as

$$
\omega_{D}=\frac{e}{2 \epsilon_{0}} \cdot \frac{n_{\text {cathode }}(r)}{\gamma^{2} \cdot B_{\text {cathode }}} .
$$

The temporal evolution frequency $\omega_{l}$ and the instability growth rate $\gamma_{l}$ of the diocotron instability are both proportional to the effective diocotron frequency $\omega_{D}(r)$ and can be expressed as [56-57]

$$
\omega_{l}=\frac{\omega_{D}}{2}\left[l\left(1-\frac{b^{2}}{d^{2}}\right)+\frac{d^{2 l}}{R^{2 l}}\left(1-\frac{b^{2 l}}{d^{2 l}}\right)\right]
$$

and

$$
\begin{aligned}
\gamma_{l}= & \frac{\omega_{D}}{2}\left\{4 \frac{b^{2 l}}{d^{2 l}}\left[1-l\left(1-\frac{b^{2}}{d^{2}}\right) \frac{d^{2 l}}{R^{2 l}}\right]\right. \\
& \left.-\left[2-l\left(1-\frac{b^{2}}{d^{2}}\right)-\frac{d^{2 l}}{R^{2 l}}\left(1-\frac{b^{2 l}}{d^{2 l}}\right)\right]^{2}\right\}^{1 / 2},
\end{aligned}
$$

respectively, where $l$ is the azimuthal mode number of the perturbation. From Eq. (10), the instability exists for $l \geq 2$ when the growth rate $\gamma_{l}$ is a real number, which means [56-57]

$$
\begin{aligned}
& 4 \frac{b^{2 l}}{d^{2 l}}\left[1-l\left(1-\frac{b^{2}}{d^{2}}\right) \frac{d^{2 l}}{R^{2 l}}\right] \\
& >\left[2-l\left(1-\frac{b^{2}}{d^{2}}\right)-\frac{d^{2 l}}{R^{2 l}}\left(1-\frac{b^{2 l}}{d^{2 l}}\right)\right]^{2} .
\end{aligned}
$$

Equation (11) shows that, for a uniform density of the hollow electron beam with an external conducting 


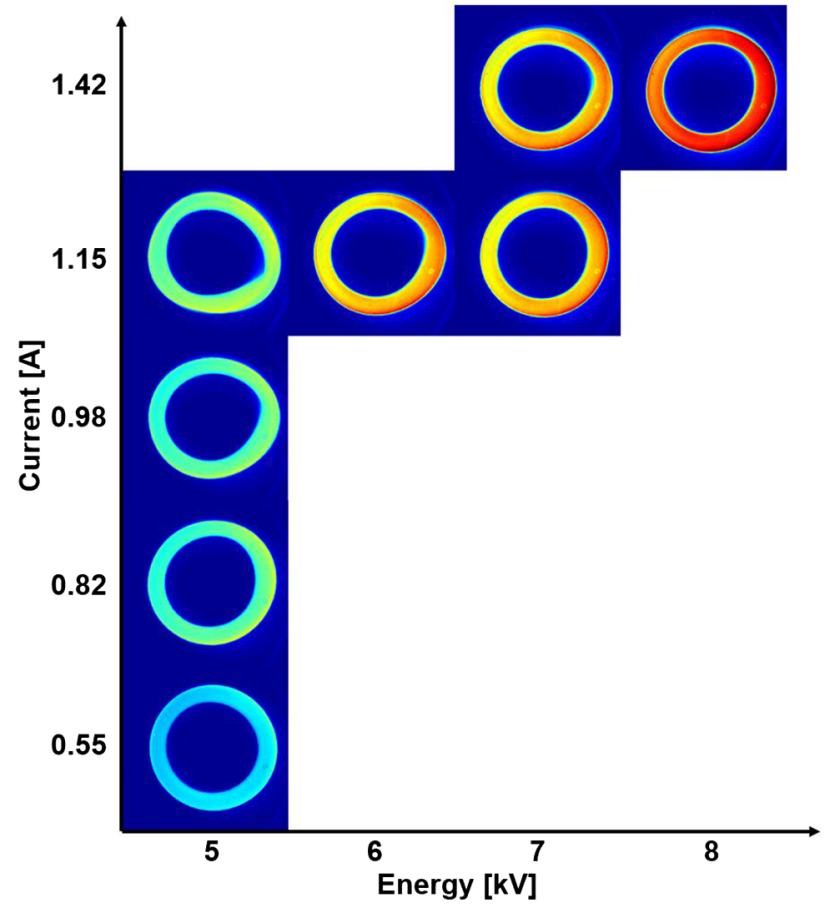

FIG. 4. Measured hollow beam profiles with different electron beam currents and energy. The color scale represents the relative intensity of the pixels with arbitrary units.

boundary, the beam stability condition depends on $l, b, d$, and $R$. During the hollow electron gun design, one can optimize the above parameters to increase the diocotron instability threshold. But the RHIC hollow cathode was designed to fit into the existing cathode holder, restricting the choice of the three geometry parameters $b, d$, and $R$.

If the vacuum pipe radius is much greater than the beam size, Eq. (11) can be simplified to [56-57]

$$
4 \frac{b^{2 l}}{d^{2 l}}>\left[2-l\left(1-\frac{b^{2}}{d^{2}}\right)\right]^{2}
$$

For a nonuniform density of the electron beam, the recent hollow beam diocotron instability study [56] shows that small azimuthally initial disturbances or the asymmetry of the electron density may lead to significant beam density distortions.

After the above theoretical estimates, the beam stability under various conditions was studied experimentally and compared with the theoretical model, as described below.

\section{B. Experimental studies}

To reduce the hollow beam profile distortions, and therefore the potential for emittance growth from a residual field in the core, profiles were recorded for different machine conditions. These include the variations in the beam density $n_{\text {cathode }}(r)$ and the magnetic field $B_{\text {cathode }}$ for the diocotron instability growth rate control and the beam energy $(\gamma)$ for the instability developing in time from the cathode to the YAG screen.

Figure 4 shows the beam profiles for different electron beam currents as a function of the electron beam energy. At a beam energy of $5 \mathrm{keV}$, distortions started to appear already at $0.82 \mathrm{~A}$ and increased at higher currents. The
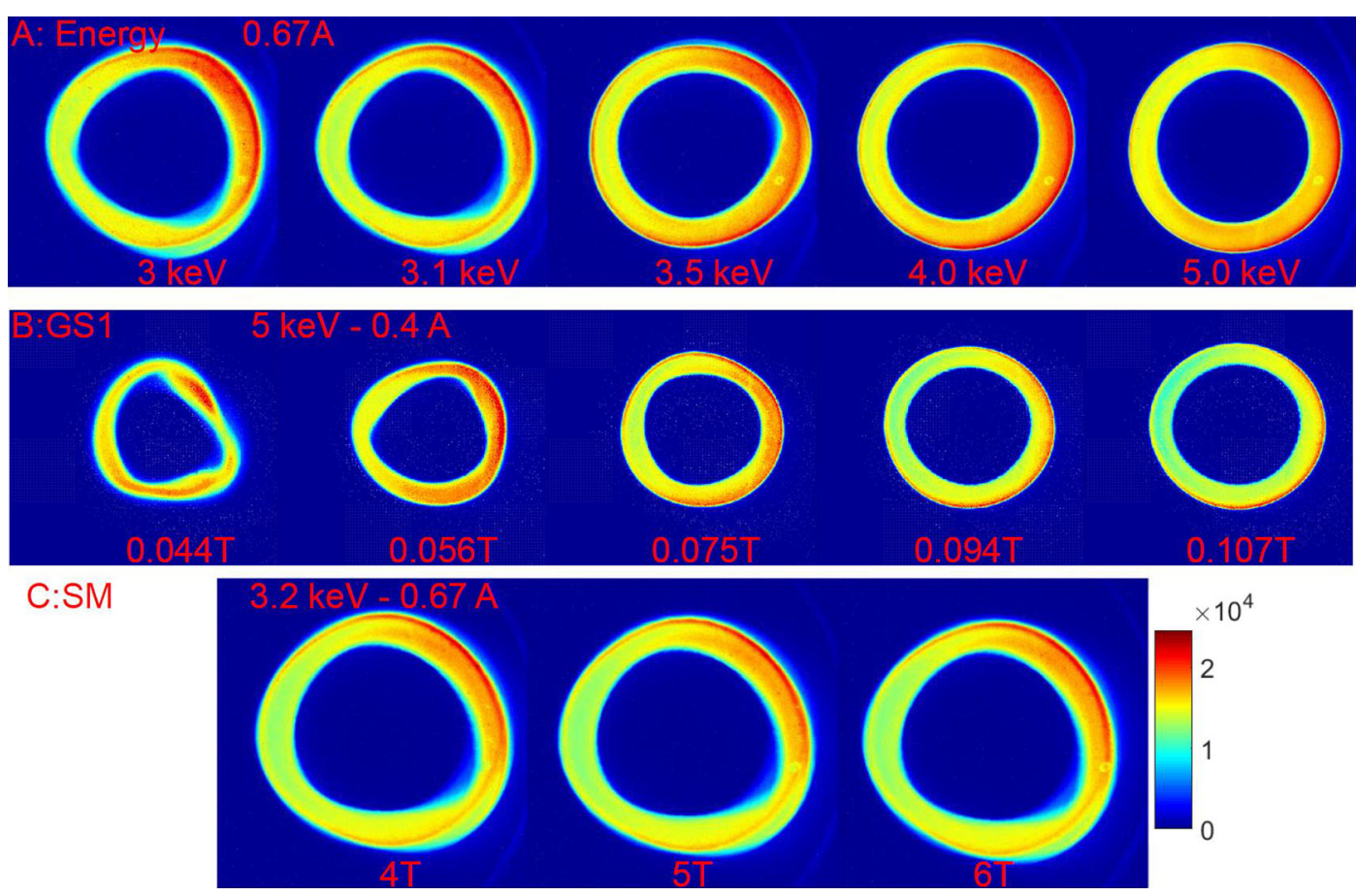

FIG. 5. Measured hollow beam profiles with different electron beam energy (a), GS1 magnetic field (b), and superconducting solenoid magnetic field (c). The color scale represents the relative intensity of the pixels with arbitrary units. 

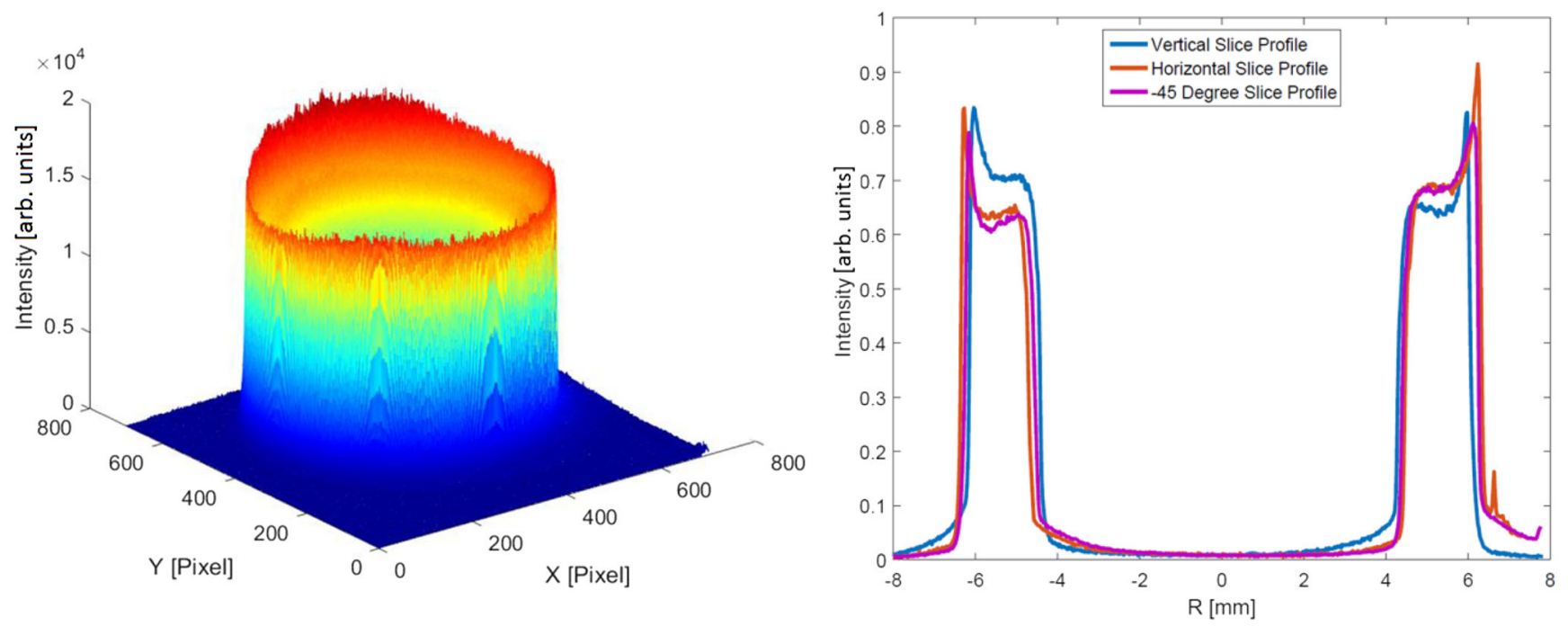

FIG. 6. Measured hollow beam profiles with 0.27 A current and $5 \mathrm{keV}$ energy.

diocotron instability can be suppressed by increasing the electron beam energy. This is shown for a current of $1.15 \mathrm{~A}$ when going from 5 to 6 to $7 \mathrm{keV}$ and at $1.42 \mathrm{~A}$ when going from 7 to $8 \mathrm{keV}$.

Figure 5(a) depicts a similar plot that demonstrates that the higher electron beam energy can suppress the diocotron instability for an electron beam current of $0.67 \mathrm{~A}$. The superconducting magnetic field was $3 \mathrm{~T}$, and the GS1 field was $0.32 \mathrm{~T}$.

For the $1.15 \mathrm{~A}-5 \mathrm{keV}$ beam image in Fig. 4 and the $0.67 \mathrm{~A}-3 \mathrm{keV}$ image in Fig. 5(a), the diocotron instability is very clear, and the hollow electron beam starts to form three clusters corresponding to the azimuthal wave number $l=3$. For the RHIC hollow electron lens, the beam inner radius is $b=4.0 \mathrm{~mm}$, the beam outer radius $d=5.5 \mathrm{~mm}$, and vacuum pipe radius $R=54.6 \mathrm{~mm}$; inequality (12) reads $0.592>0.344-$ i.e., the instability criterion is satisfied.

Figure 5(b) shows the electron beam images as a function of the GS1 magnetic field. No diocotron instability is found with the GS1 magnetic field at $0.107 \mathrm{~T}$, but distortions appear when the field is reduced. The energy was $5 \mathrm{keV}$, the current was $400 \mathrm{~mA}$, and the superconducting solenoid field was $3 \mathrm{~T}$ in Fig. 5(b).

The experiments confirmed the theoretical expectation [Eq. (8)] that the diocotron instability can be suppressed with two methods. One method is to have a higher cathode field resulting in a lower diocotron temporal evolution frequency and instability growth rate. The other is a higher beam energy resulting in less time available for the instability development, as well as a lower diocotron temporal evolution frequency and instability growth rate.

The experiments also confirm the conclusion from Eq. (8) that the magnetic fields other than at the cathode do not affect the instability. In Fig. 5(c), the superconducting solenoid magnetic field was changed from 4 to $6 \mathrm{~T}$ during the experiments, and the instability did not change.
The electron beam was $0.67 \mathrm{~A}$, the electron beam energy was $3.2 \mathrm{keV}$, and the GS1 field was $0.38 \mathrm{~T}$.

Figure 6 shows the 3D and 2D plot of the nominal profile during the experiments. From the plot, one can find that the profile of the hollow lens is close to a round ring with $16.7 \%$ peak to peak intensity variation. The higher current density at the rim comes from the geometry of the hollow cathode, the control electrode, and the anode. This has been confirmed by the hollow cathode simulation.

\section{TRANSVERSE ALIGNMENT}

The transverse alignment of a Gaussian electron with a Gaussian ion beam, including the position alignment and angle alignment, was demonstrated with an electron-backscattering detector (eBSD) [37] during the commissioning and the operation of beam-beam compensation [29,34].

As discussed in the previous section, a misalignment between the hollow electron and the ion beam could result in ion beam emittance growth in pulsed mode operation. Since the backscattered electrons were collected only above the electron beam, the feasibility of using the eBSD for the alignment needed to be demonstrated for hollow electron beam distributions.

When the electron beam interacts with the hadron beam within the superconducting magnet, backscattered electrons stream back along the magnetic field lines from the superconducting magnet to GS2 in a curved magnetic field (Fig. 1). Because of this curved magnetic field, the curvature drift velocity of the backscattered electrons is [58]

$$
\vec{v}_{R}=\frac{m v_{\|}^{2}}{q B^{2}} \cdot \frac{\vec{R}_{c} \times \vec{B}}{R_{c}^{2}},
$$

where $B$ is the magnetic field, $v_{\|}$is the transverse beam velocity, and $R_{c}$ is the radius of the trajectory. 


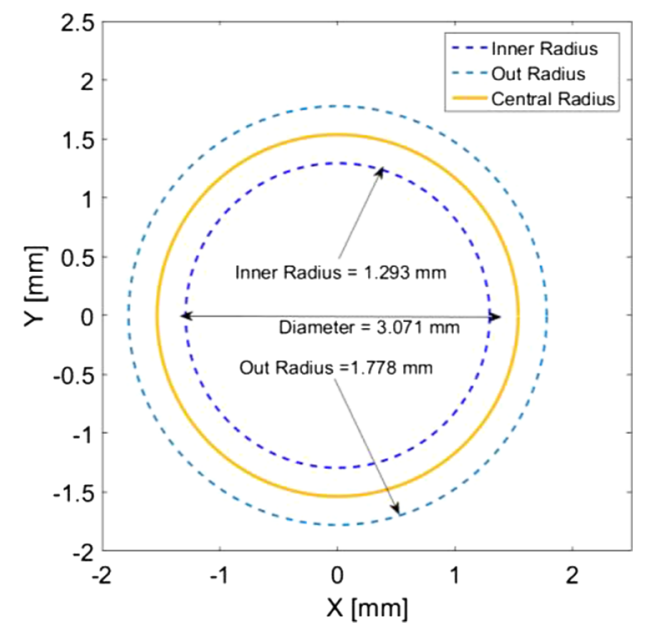

FIG. 7. Hollow electron beam geometry parameters.

The curvature drift velocity direction was designed to be vertical upwards, and the eBSD was installed above the electron beam. The asymmetric eBSD location reduces the received signal for electrons backscattered from the bottom of the hollow electron beam. Before the alignment with the eBSD, both the hollow electron beam and the hadron beam position were measured by the BPMs for the initial alignment. The hadron beam was then moved horizontally and vertically across the electron beam.

During the transverse alignment, the gun solenoid field GS1 was $0.32 \mathrm{~T}$, and the superconducting magnet field was 3.0 T. With the cathode dimensions, this yields an inner radius of $1.29 \mathrm{~mm}$, an outer radius of $1.78 \mathrm{~mm}$, and a central diameter of $3.07 \mathrm{~mm}$ inside the superconducting magnet (Fig. 7).

In the experiments, a ruthenium $(\mathrm{Ru})$ beam was used with a normalized rms emittance of $1.1-1.4 \mu \mathrm{m}$ and an energy of $100 \mathrm{GeV} /$ nucleon. This corresponds to a $0.23-0.26 \mathrm{~mm} \mathrm{Ru}$ beam size (both planes) inside the superconducting solenoid. In the scans, the hollow electron beam was kept unchanged, while the Ru beam was moved in steps of $0.2 \mathrm{~mm}$ in both the vertical and the horizontal planes by the RHIC Luminosity and IR Steering Application (LISA) application [59]. The dc current of the hollow electron beam was $40 \mathrm{~mA}$. The eBSD counting rate was recorded at the same time. Both horizontal and vertical position scans are presented in Fig. 8.

In the horizontal scan, the eBSD signal shows two peaks $(Y=1.246$ at $X=0.161 \mathrm{~mm}$ and $Y=0.902$ at $X=3.363 \mathrm{~mm}$ ). The vertical scan was carried out and is shown as the blue curve in Fig. 8. The vertical data also show two peaks $(Y=0.693$ at $X=-5.707 \mathrm{~mm}$ and $Y=$ 2.011 at $X=-2.429 \mathrm{~mm}$ ).

The distances between the horizontal and vertical peaks ( 3.20 and $3.28 \mathrm{~mm}$, respectively) are close to the calculated hollow beam central diameter of $3.07 \mathrm{~mm}$ (Fig. 7). This indicates that the Ru beam was already aligned to the center of the hollow beam with the BPMs. If the beam was off

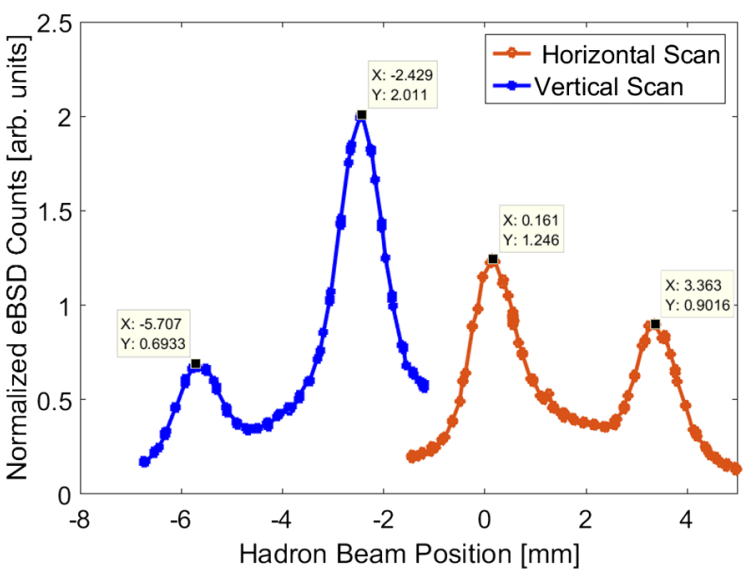

FIG. 8. Transverse beam alignment with the eBSD, with $40 \mathrm{~mA}$ dc beam.

center, the distance between the two peaks will be less than the central diameter.

In the horizontal scan, the amplitude of one eBSD peak is higher than the other, which could be caused by the nonuniform electron beam intensity, the U-shape e-beam trajectory, or an angle between the hadron and electron beam.

For the vertical plane, because the eBSD is installed above the electron beam, the amplitude difference between the two vertical peaks (the blue curve) is mainly due to the different detection efficiency for electrons backscattered from the bottom and the top of the hollow electron beam. This position-dependent detection efficiency can be calibrated and corrected, but this was not done in the present experiments. An even better method to avoid this effect is to automate the vertical detector position control so as to coordinate the motion of the detector with the motion of the beam during a scan. To account for the different Larmor radii, the displacement of the detector would be scaled up by a factor $B_{s} / B_{d}$, where $B_{s}$ is the field in the solenoid and $B_{d}$ is the field at the detector position. This system was not implemented, because the position-sensitive efficiency variations were much less significant during the normal scans of electron beams with smaller radii and Gaussian profiles.

Contributing factors to the observed asymmetry could also be the ion beam intensity decay, the nonuniformity of the e-beam intensity, or the angle between the hadron and electron beam.

The angle alignment was also checked after the position alignment, and the corresponding scans are shown in Fig. 9 for the vertical plane. The angle was changed by $\pm 0.1 \mathrm{mrad}$ to repeat the transverse position scan two more times. There is no significant difference between vertical scans for the different angle settings, which indicates a good angle alignment. The horizontal plane has a similar result. The angle alignments were carried out with a $90 \mathrm{GeV} /$ nucleon $\mathrm{Au}$ beam in 2019. 


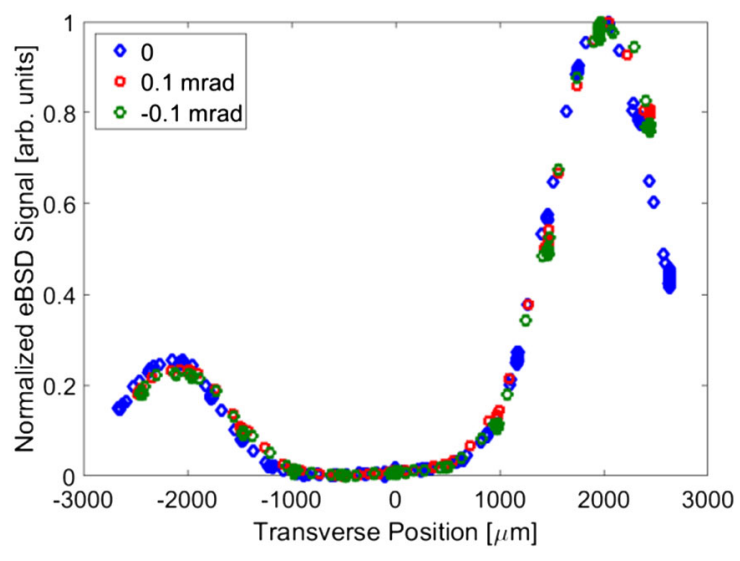

FIG. 9. Angle alignment with the eBSD.

The results successfully demonstrated that the eBSD can be used for the hollow beam alignment in both the horizontal and vertical planes.

\section{EXPERIMENTS WITH COLLIDING BEAMS}

\section{A. Experiment setup}

After demonstrating that the eBSD can be used for the hollow beam alignment, the hollow electron beam was then used for experiments with colliding beams.

To find fast halo diffusion rates while preserving the beam core, the dependences of the beam loss rate (halo), emittance (core), and beam distribution (halo and core) on the hollow electron beam parameters, the pulse pattern of the electron beam, and machine configuration were investigated.

In the experiments, two bunch trains were used: one with the hollow lens acting on it and one which was not affected by it (Fig. 10, top plot). Each bunch train had 53 bunches with five empties between them. The pulsed hollow electron beam was turned on for the affected bunch train with a $78 \mathrm{kHz}$ frequency, which is the RHIC revolution frequency.

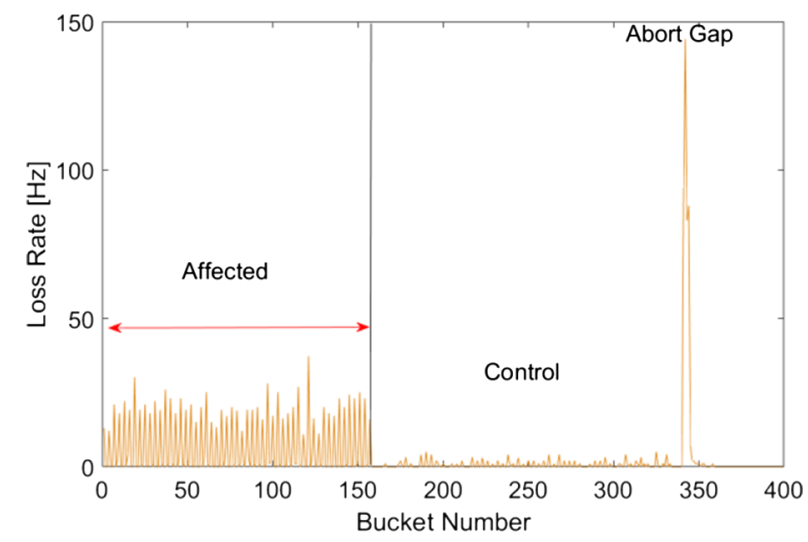

FIG. 10. Yellow bunch-by-bunch (BBB) loss rate at the collimator for bunches affected by the hollow lens and control bunches.
Three beam energies (100, 90, and $13.5 \mathrm{GeV} /$ nucleon) were used in the experiments, and the ion beam species were $\mathrm{Ru}$ (2018) and $\mathrm{Au}$ (2019). The main parameters of the ion and electron beams are shown in Table I. During the experiments, the ion beam in the yellow ring was used for colliding with the hollow electron beam. The beam in the blue ring was used to collide with the yellow beam and get a collision signal at IP8.

\section{B. Ion beam loss and e-beam parameters}

To study the ion beam loss as a function of the electron beam parameters, the hollow electron beam current and the beam size were scanned. The ion beam loss rate was measured bunch by bunch with $p-i-n$ diodes at the collimator.

The beam losses as a function of the hollow electron beam current for two different energies $(100 \mathrm{GeV} /$ nucleon for the $\mathrm{Ru}$ beam and $13.5 \mathrm{GeV} /$ nucleon for the $\mathrm{Au}$ beam) are shown in Fig. 11. The bunch-by-bunch beam loss rate for $13.5 \mathrm{GeV} /$ nucleon is shown in the left plot as an example, while the loss-rate ratio between the two bunch trains is depicted in the right plot. For the $100 \mathrm{GeV} /$ nucleon Ru beam, the electron beam inner radius was $4 \sigma$ of the rms ion beam size, and the collimator position was at $\sim 7 \sigma . \sigma$ is the rms beam size of the ion beam. For the $13.5 \mathrm{GeV} /$ nucleon beam, the inner radius was set to $3 \sigma$, and the collimator position was at $3.5 \sigma$. From these two plots, it can be concluded that the control bunches are not affected by the hollow electron beam current. For the affected bunch train, the loss rate is linearly proportional to the electron beam current.

Figure 12 depicts the average beam loss rate of the two bunch trains (left) at $13.5 \mathrm{GeV} /$ nucleon and the ratio between the loss rates of the two bunch trains (right), as a function of the electron beam inner radius expressed in units of $\sigma$.

Similar to the current scan results, there are no significant effects on the control bunches. But the beam loss rate of the affected bunch train increases as the electron beam size decreases.

For the $100 \mathrm{GeV} /$ nucleon Ru beam, the electron beam current was $270 \mathrm{~mA}$, and the collimator position was $\sim 7 \sigma$. For the $13.5 \mathrm{GeV} /$ nucleon beam, the electron beam current was $200 \mathrm{~mA}$, and the collimator position was $3.5 \sigma$.

\section{C. nth turn excitation}

The above experiments were carried out in the $78 \mathrm{kHz}$ mode, i.e., one electron-lens excitation per turn. To increase the halo removal rate, a resonant excitation pattern was tested where the electron beam was turned on every nth turn.

The average bunch-by-bunch beam loss rate is depicted in Fig. 13 for the $13.5 \mathrm{GeV} /$ nucleon ion beam, showing that the maximum beam loss rate occurs with the ninth turn excitation of the hollow electron beam for both the affected and the control bunch trains. The yellow ring tunes were $(0.235,0.227)$ for the horizontal and vertical plane, 

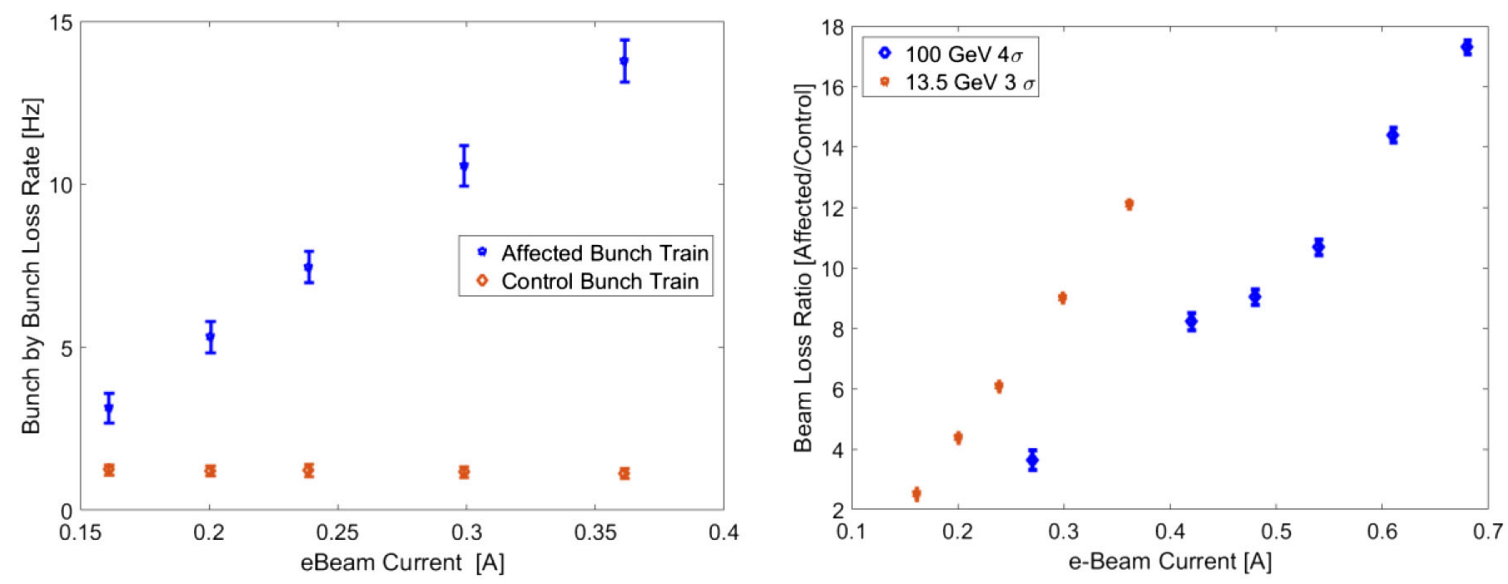

FIG. 11. Average beam loss rate measured at the collimator vs beam current. For the affected bunch train, the loss rate is proportional to the electron beam current for both ion beam energies. For the control bunch train, the loss rate is not affected by the beam current.
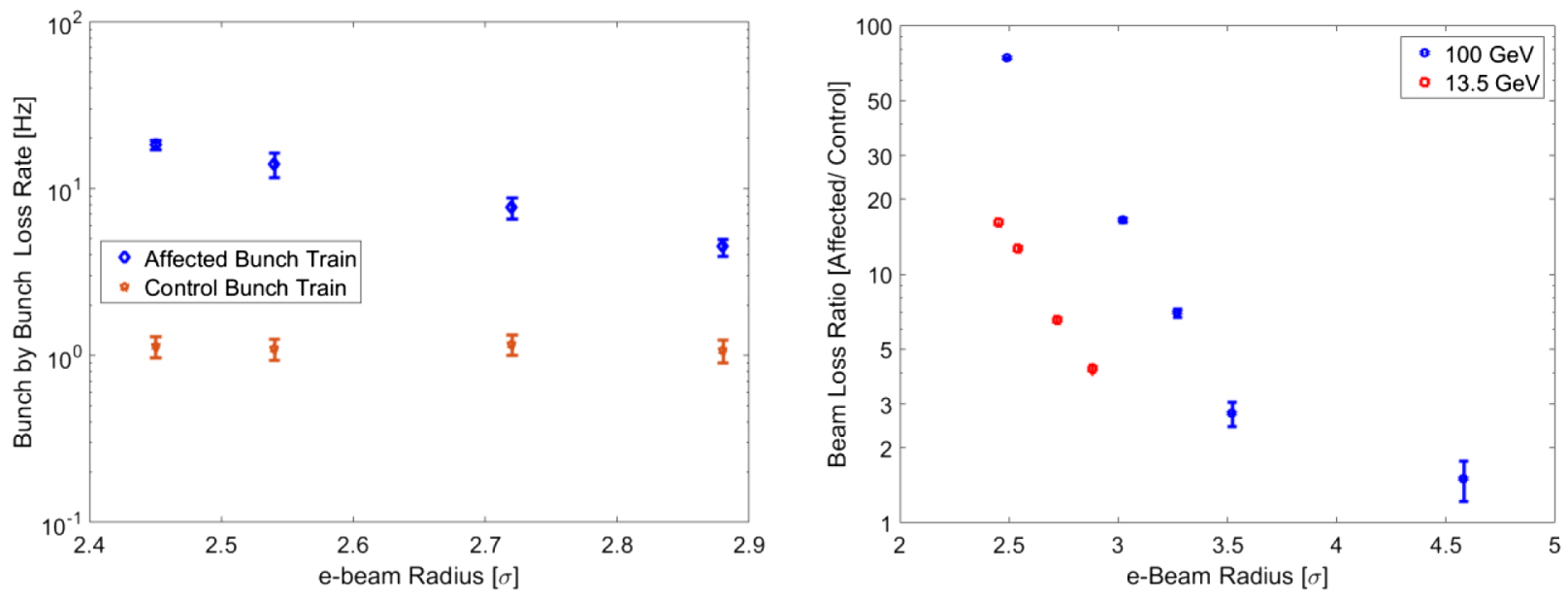

FIG. 12. Measured beam loss rate at the collimator vs beam radius. Note that the vertical scale is logarithmic for both plots.

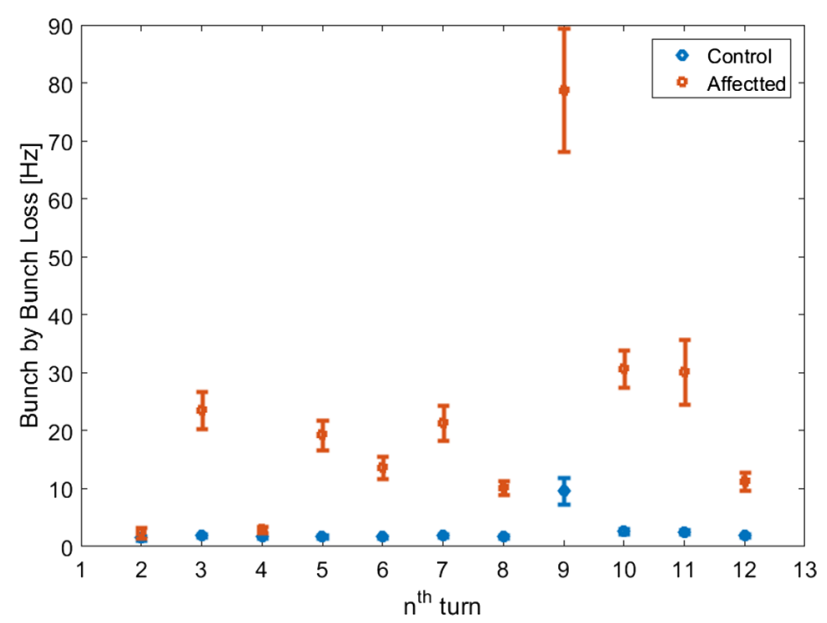

FIG. 13. Beam loss rate at the collimator vs n, where the hollow lens is turned on every nth turn. respectively, which were close to $\frac{2}{9}=0.222$. The beam loss rate is high if the RHIC lattice is operated near a resonance line.

Although the nth turn excitation may have a higher halo removal rate, the beam core and the emittance of the affected ion beam could be excited as well. Further study about this is needed.

During the excitation experiments, we found that the blue beam had significant beam loss (Fig. 14). The loss could be higher than the yellow ion beam in the other modes. It could be directly affected by the yellow hollow ebeam. During the experiments, the inner and outer radius of the hollow electron beam are 3.02 and $4.15 \mathrm{~mm}$, respectively. Both blue and yellow ion beams share the same beam pipe. The measured distance between the blue ion beam and the yellow ion beam was $8.2 \mathrm{~mm}$, which was about 2 times the outer radius of the hollow electron beam. Thus, the yellow hollow electron beam excited the blue ion beam by means of a dipole kick. 


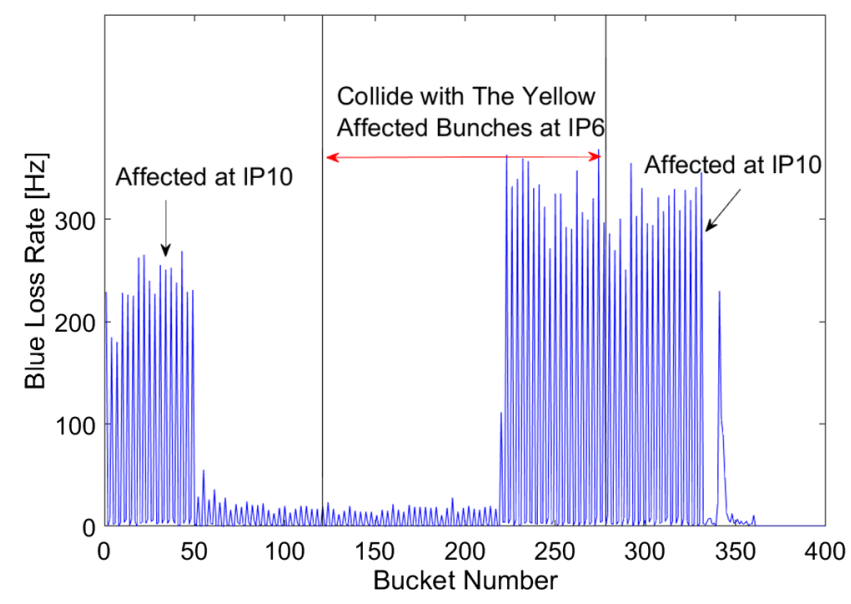

FIG. 14. Blue BBB loss rate affected by the yellow hollow lens in the nth turn excitation mode because of dipole kicks at IP10.

Meanwhile, with the ninth turn excitation, there were also some losses from the control bunch train (Fig. 13). According to the RHIC fill pattern, the yellow control bunch train was then affected from the blue through the beam-beam interaction at IP6.

From Fig. 14, the yellow affected bunch train interacted with part of the excited blue bunches (the bunches between two vertical lines with higher blue beam loss). These excited blue bunches (through the dipole kick at IP10) caused a visible loss of the yellow affected bunch train (through the beam-beam effect at IP6). The yellow control bunch train also interacted with the other part excited blue bunches. Therefore, both the affected and the control yellow bunch trains had beam losses because of the blue beam excitation at IP10.

\section{Luminosity, intensity, and emittance}

Although the transverse profile of the hollow electron beam was very close to being round, the density of the hollow beam was not uniform. The distorted hollow cylinder, as well as the electron beam bending regions in which the ion beam goes through the hollow beam, may have some effect on the ion beam intensity, rms emittance, and luminosity.

To explore these effects, the bunch-by-bunch luminosity and the bunch-by-bunch beam intensity were recorded, and the emittances averaged over the train. This experiment was done with a $90 \mathrm{GeV} /$ nucleon Au beam and a $516 \mathrm{~mA}$ and $5 \mathrm{keV}$ hollow electron beam. With these parameters, the hollow beam was round (Fig. 5). The operational mode was the pulsed mode with $78 \mathrm{kHz}$ (one excitation per turn). The average beta function at the center of the electron lens was $7.35 \mathrm{~m}$.

The top plot in Fig. 15 depicts the average (horizontal and vertical) emittance of the affected bunch train. Before the radius scan, the emittance increases because of intrabeam scattering (IBS) and a nonlinear effect including the beam-beam interaction at IP6. With a reduction of the hollow electron beam inner radius from $4.3 \sigma$ to $4.1 \sigma$, the

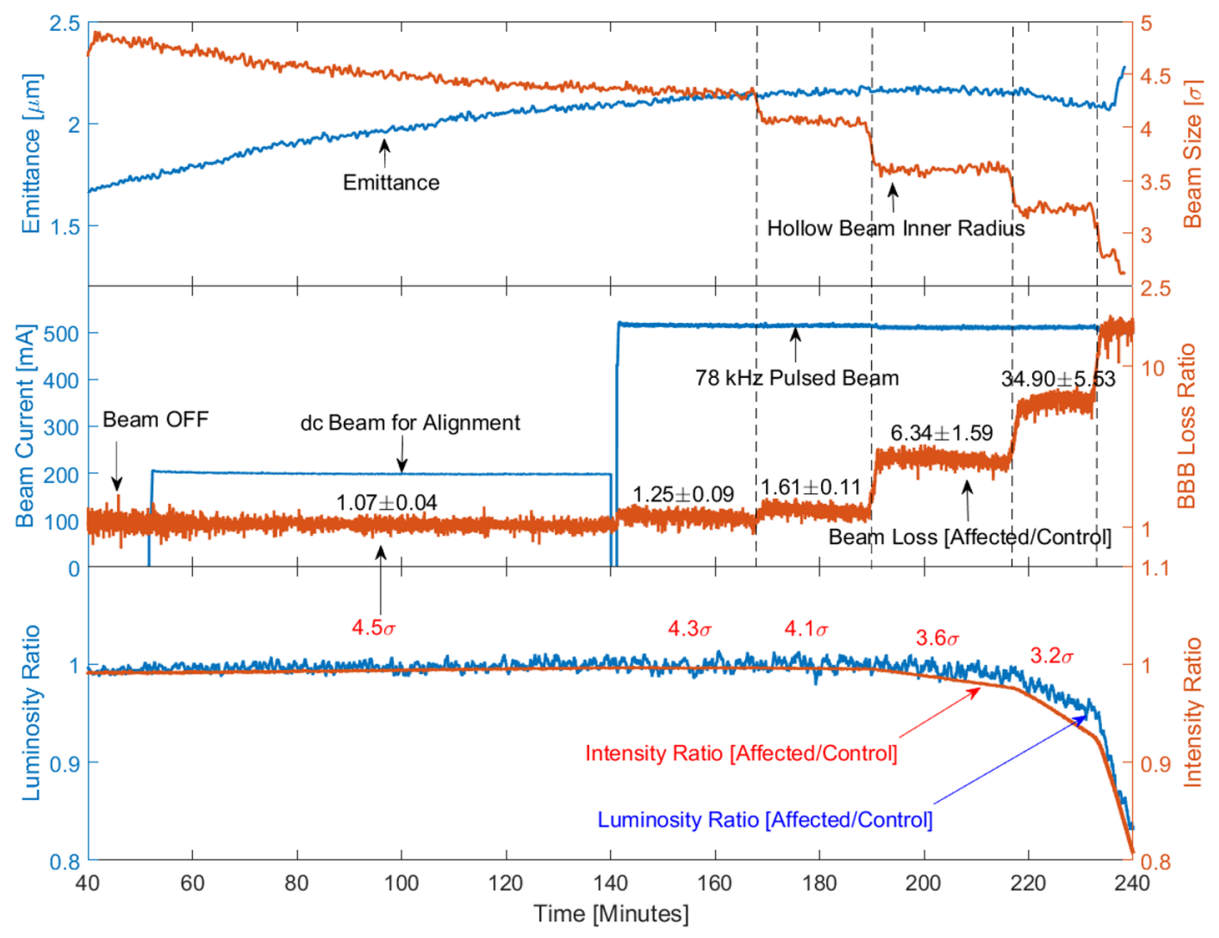

FIG. 15. Overview of the radius scan experiments. The top plot shows the average (horizontal and vertical) emittance of the affected bunch train. The middle plots are the electron beam current and beam BBB loss ratio during experiments. The bottom plot shows the normalized average intensity and the average luminosity. 
emittance growth rate was reduced. At $3.6 \sigma$, it became flat, because the transverse beam tail was removed by the hollow lens. After the last step $(2.7 \sigma)$, there was a $0.2 \mu \mathrm{m}$ (10\%) emittance jump, indicating a significant effect on the core.

The middle plot shows the electron beam current during the alignment and the radius scan experiments. The bunchby-bunch beam loss ratio (log scale) between the affected bunch train and the control bunch train is also presented. The beam loss ratio has a clear step when the beam was changed from dc mode to pulsed mode as well as every time the beam inner radius changes. Both bunch trains were affected by the hollow beam during the alignment with the dc mode beam. We can see the losses of the affected train are larger than the control bunch train by about $7 \%$ during the beam alignment $(4.5 \sigma)$, for which the reasons are still under investigation. When the hollow electron beam interacted only with the affected bunch train in the pulsed mode, the losses from the affected train are $25 \%$ and $61 \%$ higher than the control bunch train for $4.3 \sigma$ and $4.1 \sigma$ inner radius, respectively.

The bottom plot shows the normalized average bunchby-bunch intensity and the average bunch-by-bunch luminosity (averaged over $32 \mathrm{~s}$ ) for the two bunch trains. It contains the intensity and luminosity ratio (affected bunch train/control bunch train) when the hollow beam inner radius was changed from $4.3 \sigma$ to $3.2 \sigma$. Each step was kept for about $20 \mathrm{~min}$. The last scan (below $3 \sigma$ ) is not considered, because the beam core was significantly affected. The loss rate during the last step was $120 \% / \mathrm{hr}$.

In the bottom plot in Fig. 15, the intensity and luminosity are linearly fitted for each step during the radius scan. The slopes of the fits are shown in Fig. 16, containing the gradients of the two ratios (luminosity and intensity), as a function of the beam radius.

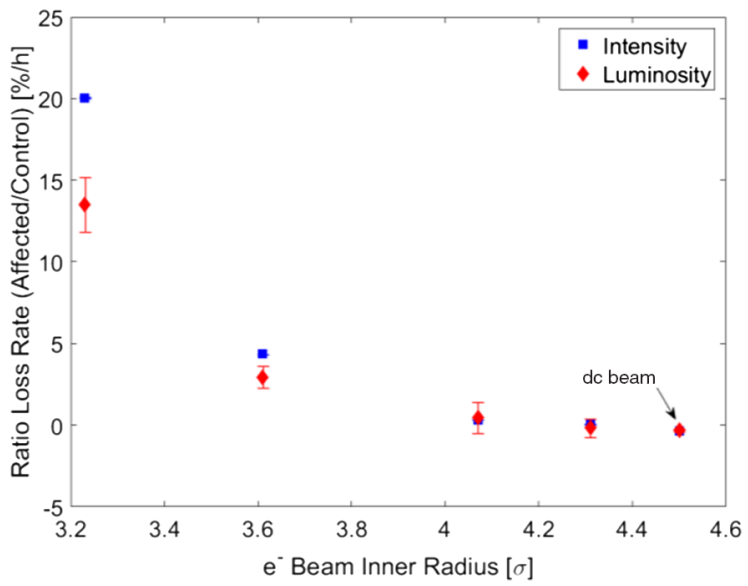

FIG. 16. The slope (absolute value) of the intensity and luminosity ratio of the two bunch trains as a function of the hollow beam inner radius. The error bar is from the fitting error.
There is always a luminosity reduction with an intensity reduction. With the inner hollow beam radius of $4.3 \sigma$, there are clear bunch-by-bunch beam losses, while there are no clear beam intensity and luminosity losses, the measurements of which may not be sensitive to such a small variation of total intensity. With the smaller inner beam size, there is a clear difference between intensity and luminosity loss rate.

Assuming Gaussian profiles in all dimensions and both blue and yellow have the same beam size on both planes, the ion beam intensity ratio between a hollow electron bean truncated and a Gaussian beam can be expressed as

$$
\frac{N_{H}}{N_{G}}=\left(1-e^{-\left(R_{C}^{2} / 2 \sigma^{2}\right)}\right),
$$

where $R_{c}$ is the inner radius of the hollow electron beam and $\sigma$ is the rms beam size. It is assumed that all particles with a position larger than the beam inner radius will lose immediately. Therefore, the ion beam intensity loss ratio is expressed as

$$
\frac{\Delta N}{N_{G}}=e^{-\left(R_{C}^{2} / 2 \sigma^{2}\right)}
$$

While for the luminosity it has an integral of $e^{-\left(r^{2} / \sigma^{2}\right)}$ component [60] for a round Gaussian beam, the luminosity loss ratio is written as

$$
\frac{\Delta L}{L_{G}}=e^{-\left(R_{C}^{2} / \sigma^{2}\right)} .
$$

Therefore, the intensity loss ratio is always higher than the luminosity loss ratio. This can be qualitatively verified in Fig. 16, although Eqs. (15) and (16) are calculated for a static state while it was a dynamic state during the experiments.

The intensity and luminosity ratio loss rate with the dc mode hollow electron beam $(4.5 \sigma)$ are also shown in the plot. The luminosity ratio loss rate is close to the one with the pulsed hollow beam on $(4.3 \sigma)$, which indicates that the hollow e-lens could be operated without significantly affecting the beam core or luminosity with the inner beam size of $4.3 \sigma$. With a smaller inner radius of $3.6 \sigma$, the luminosity and beam loss are clearly affected.

\section{SUMMARY AND DISCUSSTION}

Experiments with a hollow electron lens were carried out in the RHIC to assess the halo removal rate and detrimental effects on the beam core. A hollow lens can assist in beam collimation in existing or future hadron rings including the HL-LHC, Electron Ion Collider (EIC), FCC-hh, or SPPC.

The design of the RHIC hollow electron lens was presented. Distortions of the hollow electron beam profile were discussed, including the reasons for the distortions 
and ways to reduce them. Improvements were demonstrated experimentally resulting in a more symmetric hollow beam, which will have a smaller impact on the ion beam core.

The alignment of the hollow electron beam with the ion beam was demonstrated with the backscattered electron detector, which had previously been used for Gaussian beams. These two achievements reduce the possibility of emittance growth.

For the pulsed operation, the U-shape design of the RHIC lenses still results in a net kick experienced by the beam core, because the entrance and exit kicks do not cancel each other, which could result in additional emittance growth during the experiments. In the HL-LHC, thanks to an S-shape design, this effect will not be present.

With the hollow lens pulsed beam (turned on every turn for the affected bunch train), the halo removal rate and effect on the emittance were assessed for different currents and radii. For the affected bunch train, the loss rate is proportional to the electron beam current with the same hollow electron beam inner radius, while the beam loss rate of the affected bunch train increases as the inner electron beam size decreases.

The removal rate of the ion beam halo was also experimentally investigated with an excitation every nth turn. The halo removal rate can be increased with a resonant excitation every ninth turn, but the core emittance may simultaneously be affected.

The experiments determined that the hollow electron beam has no significant effect on the luminosity with a $4.3 \sigma$ inner beam radius and with a $16.7 \%$ measured peak-to-peak hollow electron beam intensity variation at the YAG screen. In the overlap region, the average beam density variation should not exceed $10 \%$ to avoid visible emittance growth caused by a hollow electron beam. This estimate is based on the diocotron instability along the beam trajectory, which means the electron beam density variation inside the superconducting magnet should be smaller than at the YAG screen.

With an inner radius of $3.6 \sigma$ and $3.2 \sigma$, some significantly higher losses from the affected train by factor of 6.3 and 34.9 were found, respectively. This can be an indication that the ion beam core was affected too much, and the luminosity decreased. Finally, with an inner radius of $2.7 \sigma$, the losses of the affected trains are a factor of 300 larger, indicating once more that the beam core was affected. No emittance blowup was found with an inner electron beam radius down to a $3.2 \sigma$.

\section{ACKNOWLEDGMENTS}

The work was supported by many colleagues in the Collider-Accelerator Department at Brookhaven National Laboratory. The authors appreciate valuable help and discussions with the CAD staff members, in particular Y. Luo, S. Keys, V. Schoefer, R. Than, and T. C. Shrey. Some authors are supported by the HL-LHC project. This work was supported by the U.S. Department of Energy under Contract No. DE-SC0012704. This manuscript has been also authored by Fermi Research Alliance, LLC under Contract No. DE-AC02-07CH11359 with the U.S. Department of Energy, Office of Science, Office of High Energy Physics.

[1] O. V. Arkhipov, L. V. obyleva, V. B. Brudanin et al., Linear induction accelerator of the hollow electron beam, in Proceedings of the 1990 8th International Conference on High-Power Particle Beams, Novosibirsk (1990), pp. 809-814, https://ieeexplore.ieee.org/document/ 6396364.

[2] N. Jain, T. M. Antonsen, Jr., and J. P. Palastro, Positron Acceleration by Plasma Wakefields Driven by a Hollow Electron Beam, Phys. Rev. Lett. 115, 195001 (2015).

[3] G.-B. Zhang, M. Chen, and C. B. Schroeder et al., Acceleration and evolution of a hollow electron beam in wakefields driven by a Laguerre-Gaussian laser pulse, Phys. Plasmas 23, 033114 (2016).

[4] A. Pathak and S. Krishnagopal, Improved free-electron laser stability and performance using a hollow electron beam, Phys. Rev. Accel. Beams 21, 070702 (2018).

[5] V. Parkhomchuk, Comparison of the hollow electron beam devices and electron heating, in Proceedings of $\mathrm{COOL}$ 2007 (workshop on beam cooling and related topics), Bad Kreuznach, Germany (2007), p. 64, TUM1I03, http:// accelconf.web.cern.ch/AccelConf/c107/papers/tum1i03.pdf.

[6] Y.S. Derbenev, Advanced optical concepts for electron cooling, Nucl. Instrum. Methods Phys. Res., Sect. A 441, 223 (2000).

[7] G. X. Xia, J. W. Xia, J. C. Yang, W. Liu, J. X. Wu, X. J. Yin, H. W. Zhao, and B.W. Wei, Impact on electron velocity of hollow electron beam in HIRFL-CSR e-cooler system, Nucl. Instrum. Methods Phys. Res., Sect. A 508, 239 (2003).

[8] F. Krienen and W. B. Herrmannsfeldt, Proposal for hollow cathode electron gun for electron cooling, Report No. SLAC-PUB-3445, 1984.

[9] L. R. Prost and P. Derwent, The state of the art in hadron beam cooling, Report No. FERMILAB-CONF-08-311-AD [arXiv:0811.3025].

[10] A. Bubley, A. Goncharov, A. Ivanov et al., The electron gun with variable beam profile for optimization of the electron cooling, in Proceedings of the 8th European Particle Accelerator Conference, Paris, 2002 (EPAC 2002), https://accelconf.web.cern.ch/AccelConf/e02/ PAPERS/WEPRI049.pdf.

[11] V. V. Parkhomchuk, Development of a new generation of coolers with a hollow electron beam and electrostatic bending, AIP Conf. Proc. 821, 249 (2006).

[12] V. Shiltsev, On Possible Use of Electron Lenses in LHC, in Proceedings of the Third CARE-HHH-APD Workshop (LHC-LUMI-06), Valencia, Spain (2006), p. 92, CERN-2007-002, http://care-hhh.web.cern.ch/ CARE-HHH/LUMI-06/Proceedings/IR\%20Upgrade\% 20II/LHCelenses_Shiltsev_rev.pdf. 
[13] G. Stancari, V. Previtali, A. Valishev, R. Bruce, S. Redaelli, A. Rossi, and B. S. Ferrando, Conceptual design of hollow electron lenses for beam halo control in the Large Hadron Collider, Technical Report No. FERMILAB-TM-2572APC, CERN-ACC-2014-0248 (Fermilab and CERN, 2015) [arXiv:1405.2033].

[14] S. Redaelli, A. Bertarelli, R. Bruce et al., Plans for deployment of hollow electron lenses at the LHC for enhanced beam collimation, in Proceedings of the 6th International Particle Accelerator Conference (IPAC2015), Richmond, VA, USA (JACoW, Geneva, Switzerland, 2015), WEBB1, https://doi.org/10.18429/JACoW-IPAC2015-WEBB1.

[15] https://indico.cern.ch/event/648237/timetable/\#20171019 .detailed.

[16] G. Stancari, A. Valishev, G. Annala et al., Collimation with Hollow Electron Beams, Phys. Rev. Lett. 107, 084802 (2011).

[17] V.D. Shiltsev, Electron Lenses for Super-Colliders (Springer, New York, 2016).

[18] G. Stancari, New methods of particle collimation in colliders, arXiv:1110.0144.

[19] G. Stancari et al., Development of hollow electron beams for proton and ion collimation, in Proceedings of the 1st International Particle Accelerator Conference (IPAC10), Kyoto, Japan, 2010, TUPEB076, p. 1698 (Report No. FERMILAB-CONF-10-182-AD-APC), https://arxiv.org/ftp/ arxiv/papers/1202/1202.0252.pdf.

[20] G. Stancari, Calculation of the transverse kicks generated by the bends of a hollow electron lens, Technical Report No. FERMILABFN-0972-APC, Fermilab, 2014 [arXiv:1403.6370].

[21] Y. H. Jo, J. S. Kim, G. Stancari, M. Chung, and H. J. Lee, Control of the diocotron instability of a hollow electron beam with periodic dipole magnets, Phys. Plasmas 25, 011607 (2018).

[22] S. Li and G. Stancari, Characterization of an electron gun for hollow electron beam collimation, Report No. FERMILAB-TM-2542-APC.

[23] M. Fitterer, G. Stancari, A. Valishev, and S. Redaelli, Simulation study of hollow electron beam collimation in HL-LHC, Technical Report No. FERMILAB-TM-2636AD, Fermilab, 2016.

[24] M. Fitterer, G. Stancari, A. Valishev, R. Bruce, S. Redaelli, B. M. Salvachua Ferrando, D. Valuch, G. Valentino, J. Wagner, and $\mathrm{C}$. $\mathrm{Xu}$, Effect of a resonant excitation on the evolution of the beam emittance and halo population, Technical Report No. CERNACC- NOTE-2018-0007, CERN, 2018.

[25] M. Fitterer, G. Stancari, A. Valishev, S. Redaelli, and D. Valuch, Resonant and random excitations on the proton beam in the Large Hadron Collider for active halo control with pulsed hollow electron lenses, Report No. FERMILAB-PUB-18-084-AD-APC [arXiv:1804.07418].

[26] M. Fitterer, G. Stancari, A. Valishev, R. De Maria, S. Redaelli, K. Sjobak, and J. F. Wagner, Implementation of hollow electron lenses in SIXTRACK and first simulation results for the HLLHC, in Proceedings of the 8th International Particle Accelerator Conference (IPAC2017), Copenhagen, Denmark (JACoW, Geneva, Switzerland, 2017), pp. 3795-3798, https://doi.org/ 10.18429/JACoW-IPAC2017-THPAB041.
[27] M. Fitterer, G. Stancari, and A. Valishev, Effect of pulsed hollow electron-lens operation on the proton beam core in LHC, Technical Report No. FERMILAB-TM-2635-AD, Fermilab, 2016.

[28] V. Moens, Experimental and numerical studies on the proposed application of hollow electron beam collimation for the LHC at CERN, Report No. FERMILAB-MASTERS-2013-02, CERN-THESIS-2013-126, https://doi .org/10.2172/1128148.

[29] W. Fischer, X. Gu, Z. Altinbas et al., Operational Head-on Beam-Beam Compensation with Electron Lenses in the Relativistic Heavy Ion Collider, Phys. Rev. Lett. 115, 264801 (2015).

[30] Y. Luo, W. Fischer, N. P. Abreu, X. Gu, A. Pikin, and G. Robert-Demolaize, 6-D weak-strong simulation of head-on beam-beam compensation in the Relativistic Heavy Ion Collider, Phys. Rev. Accel. Beams 15, 051004 (2012).

[31] X. Gu, F. Z. Altinbas, E. Beebe et al., The electron lens test bench for the Relativistic Heavy Ion Collider at Brookhaven National Laboratory, Nucl. Instrum. Methods Phys. Res., Sect. A 743, 56 (2014).

[32] W. Fischer, E. C. Aschenauer, Z. Altinbas et al., RHIC performance with stochastic cooling for ions and head-on beam-beam compensation for protons, in 7th International Particle Accelerator Conference (IPAC2016), 2016, BEXCO, Busan Korea, WEZA01, pp. 2055-2060, http:// accelconf.web.cern.ch/AccelConf/ipac2016/papers/weza01 .pdf.

[33] X. Gu, W. Fischer, Z. Altinbas et al., Electron lenses for head-on beam-beam compensation in RHIC, Phys. Rev. Accel. Beams 20, 023501 (2017).

[34] W. Fischer, X. Gu, K. A. Drees et al., Compensation of head-on beam-beam induced resonance driving terms and tune spread in the Relativistic Heavy Ion Collider, Phys. Rev. Accel. Beams 20, 091001 (2017).

[35] A Abada et al., FCC-hh: The Hadron Collider, Eur. Phys. J. Spec. Top. 228, 755 (2019).

[36] J. Tang et al., Concept for a future super proton-proton Collider, arXiv:1507.03224.

[37] P. Thieberger, Z. Altinbas, C. Carlson et al., High energy Coulomb-scattered electrons for relativistic particle beam diagnostics, Phys. Rev. Accel. Beams 19, 041002 (2016).

[38] A. I. Pikin, J. G. Alessi, M. Anerella et al., Structure and design of the electron lens for RHIC, in Proceedings of the 24th Particle Accelerator Conference, PAC2011, New York, NY, USA (IEEE, New York, 2011), pp. 23092311, https://accelconf.web.cern.ch/accelconf/PAC2011/ papers/thp100.pdf.

[39] X. Gu, A. Pikin, M. Okamura, W. Fischer, Y. Luo, R. Gupta, J. Hock, and D. Raparia, Designing a beam transport system for RHIC's electron lens, in Proceedings of the 24th Particle Accelerator Conference, PAC-2011, New York, 2011 (IEEE, New York, 2011), pp. 1205-1207, https://pdfs.semanticscholar.org/debb/ 4b88862c455ba475d30aa94f235cfd3edc53.pdf.

[40] R. Gupta, M. Anerella, W. Fischer et al., Magnetic design of e-lens solenoid and corrector system for RHIC, in Proceedings of the 24th Particle Accelerator Conference, PAC-2011, New York, 2011 (IEEE, New York, 2011), 
pp. 1130-1132, https://accelconf.web.cern.ch/accelconf/ PAC2011/papers/tup164.pdf.

[41] T. A. Miller, J. Aronson, D. M. Gassner et al., RHIC electron-lens beam profile monitoring, in Proceedings of the 5th and last Beam Instrumentation Workshop (BIW2012), Newport News, VA, USA (2012), pp. 213215, TUPG039, https://accelconf.web.cern.ch/accelconf/ BIW2012/papers/tupg039.pdf.

[42] T. Miller, M. Costanzo, W. Fischer et al., Beam profile measurements in the RHIC electron lens using a pinhole detector and YAG screen, in Proceedings of $3 \mathrm{rd}$ International Beam Instrumentation Conference (IBIC 2014), Monterey, CA, USA (2014), pp. 59-63, MOPF08, http://accelconf.web.cern.ch/AccelConf/IBIC2014/papers/ mopf08.pdf.

[43] http://www.fieldp.com/.

[44] X. Huang, J. Corbett, J. Safranek, and J. Wu, An algorithm for online optimization of accelerators, Nucl. Instrum. Methods Phys. Res., Sect. A 726, 77 (2013).

[45] http://multimedia.3m.com/mws/media/9511970/3m-tungstendispenser-cathodes-data-sheet.pdf.

[46] A. W. Chao and M. Tigner, Handbook of Accelerator Physics and Engineering (World Scientific, Singapore, 1999), p. 100.

[47] C. D. Child, Discharge from Hot CaO, Phys. Rev. 32, 492 (1911).

[48] I. Langmuir, The Effect of Space Charge and Initial Velocities on the Potential Distribution and Thermionic Current between Parallel Plane Electrodes, Phys. Rev. 21, 419 (1923).
[49] M. Reiser, Theory and Design of Charged Particle Beams (Wiley, New York, 1994).

[50] H. F. Webster, Breakup of hollow electron beams, J. Appl. Phys. 26, 1386 (1955).

[51] C. C. Cutler, Instability in hollow and strip electron beams, J. Appl. Phys. 27, 1028 (1956).

[52] R. H. Levy, Diocotron instability in a cylindrical geometry, Phys. Fluids 8, 1288 (1965).

[53] W. Knauer, Diocotron instability in plasmas and gas discharges, J. Appl. Phys. 37, 602 (1966).

[54] W. Roy Gould, Dynamics of non-neutral plasmas, Phys. Plasmas 2, 2151 (1995).

[55] R. C. Davidson, Physics of Nonneutral Plasmas (AddisonWesley, New York, 1990).

[56] V. V. Mikhailenko, J. Seok Kim, Y. Jo, V. S. Mikhailenko, and H. J. Lee, Non-modal analysis of the diocotron instability for cylindrical geometry with conducting boundary, Phys. Plasmas 21, 052105 (2014).

[57] V. V. Mikhailenko, H. J. Lee, V.S. Mikhailenko, and N. A. Azarenkov, Non-modal analysis of the diocotron instability: Cylindrical geometry, Phys. Plasmas 20, 042101 (2013).

[58] F. F. Chen, Introduction to Plasma Physics (Wiley, New York, 1994).

[59] A. Drees and T. D'Ottavio, Luminosity optimization using automated IR steering at RHIC, in Proceedings of the 9th European Particle Accelerator Conference, Lucerne, 2004 (EPS-AG, Lucerne, 2004), p. 911, MOPLT163.

[60] W. Herr and B. Muratori, Concept of luminosity, https://cds .cern.ch/record/941318/files/p361.pdf. 